\title{
Pediatric moyamoya MRI score: an imaging-based scale to predict outcomes in surgically treated pediatric patients with moyamoya
}

\author{
Joseph H. Garcia, BS, Ramin A. Morshed, MD, ${ }^{1}$ Ethan A. Winkler, MD, PhD, ${ }^{1}$ Yi Li, MD, ${ }^{2}$ \\ Christine K. Fox, MD, MAS, ${ }^{3}$ Heather J. Fullerton, MD, MAS, ${ }^{3}$ Caleb Rutledge, MD, ${ }^{1}$ \\ Angad S. Beniwal, BS, ${ }^{1}$ Michael T. Lawton, MD, ${ }^{1,4}$ Adib A. Abla, MD, ${ }^{1}$ Nalin Gupta, MD, PhD, $, 1,5$ and \\ Steven W. Hetts, MD²

\begin{abstract}
'Department of Neurological Surgery, University of California, San Francisco; '2Department of Radiology and Biomedical Imaging, University of California, San Francisco; ${ }^{3}$ Pediatric Stroke and Cerebrovascular Disease Center, Department of Neurology, University of California, San Francisco; and ${ }^{5}$ Department of Pediatrics, University of California, San Francisco, California; and ${ }^{4}$ Department of Neurosurgery, Barrow Neurological Institute, Phoenix, Arizona
\end{abstract}

\begin{abstract}
OBJECTIVE Moyamoya is a progressive arteriopathy that predisposes patients to stroke due to stenosis of the intracranial internal carotid arteries and their proximal branches. Despite the morbidity caused by this condition, the ability to accurately predict prognosis for individual patients remains challenging. The goal of this study was to develop a systematic scoring method based on parenchymal findings on preoperative brain MRI to predict long-term outcomes for surgically treated pediatric patients with moyamoya.

METHODS A retrospective surgical cohort of pediatric patients ( $\leq 18$ years of age at the time of the initial surgery) with moyamoya from a single center were studied. Radiological variables with existing correlations between outcomes in moyamoya or other vascular diseases were chosen to score preoperative MRI based on easily defined parenchymal findings that could be rapidly assessed and used to make a numeric score. Calculated scores were correlated with clinical outcome measures using the Pearson correlation coefficient and area under the receiver operating characteristic curve (AUROC).
\end{abstract}

RESULTS A total of 35 children with moyamoya disease or moyamoya syndrome were included in the study, with a median follow-up time of 2.6 years from the time of surgery. The pediatric moyamoya MRI score (PMMS) consists of ischemic changes $(0-2 ; 0=$ none, $1=$ focal, $2=$ diffuse), encephalomalacia $(0-2 ; 0=$ none, $1=$ focal, $2=$ diffuse $)$, and hemorrhage $(0-1 ; 0=$ not present, $1=$ present). PMMSs were highly correlated with pediatric modified Rankin Scale scores at the last follow-up ( $r=0.7,95 \% \mathrm{Cl} 0.44-0.84 ; p<0.001$ ) as a six-point scale, and when dichotomized (AUROC $=0.85$ ).

CONCLUSIONS The PMMS was found to be a simple tool based on preoperative MRI data that could be quickly and easily calculated and correlated with disability. This scoring method may aid future development of predictive models of outcomes for children with moyamoya disease and moyamoya syndrome.

https://thejns.org/doi/abs/10.3171/2021.6.FOCUS21283

KEYWORDS moyamoya disease; moyamoya syndrome; pediatric; stroke; bypass surgery; magnetic resonance imaging

$\mathrm{M}$ OYAMOYA disease is a cerebrovascular condition that predisposes affected patients to stroke due to progressive stenosis of the intracranial internal carotid arteries and their proximal branches. The incidence of moyamoya disease is highest in east Asia, and familial forms account for roughly $15 \%$ of patients. ${ }^{1}$
Moyamoya disease has several unique clinical features, which include two peaks of age distribution at 5 years and at roughly 40 years. Patients with characteristic moyamoya obliterative vasculopathy plus associated conditions are categorized as having moyamoya syndrome., ${ }^{1,2}$

In recent years, it has become increasingly clear that

ABBREVIATIONS AUROC $=$ area under the receiver operating characteristic curve; $\mathrm{MCA}=$ middle cerebral artery; $\mathrm{mRS}=$ modified Rankin Scale; PMMS = pediatric moyamoya MRI score; PSOM = pediatric stroke outcomes measure; STA = superficial temporal artery; SWI = susceptibility weighted imaging; TIA = transient ischemic attack. SUBMITTED May 1, 2021. ACCEPTED June 23, 2021.

INCLUDE WHEN CITING DOI: 10.3171/2021.6.FOCUS21283. 
TABLE 1. Individual components of the PMMS

\begin{tabular}{|c|c|}
\hline & Score \\
\hline \multirow{3}{*}{ Ischemia } & $0=$ none \\
\hline & $1=$ focal \\
\hline & $2=$ diffuse ${ }^{*}$ \\
\hline \multirow{3}{*}{ Encephalomalacia } & $0=$ none \\
\hline & 1 = focal \\
\hline & $2=$ diffuse ${ }^{*}$ \\
\hline \multirow{2}{*}{ Hemorrhage } & $0=$ none \\
\hline & $1=$ present \\
\hline
\end{tabular}

* Diffuse changes were defined as bilateral or spanning multiple vascular territories.

moyamoya encompasses a broad spectrum of distinct underlying processes rather than a single, homogeneous disease. Radiological assessment is not straightforward, and clinical studies have revealed poor reproducibility among physicians in applying this diagnostic label., ${ }^{2,3}$ In children, consistent diagnosis is especially important, as it may mitigate devastating long-term morbidity. ${ }^{4}$

A current major deficiency in the understanding of moyamoya is the ability to accurately predict prognosis for individual patients. ${ }^{3,4}$ Surgical therapies for moyamoya are effective in reducing the long-term risk of stroke but are associated with potentially significant complications, including stroke. Current clinical practice relies on an imperfect combination of clinical history, angiographic staging, and a variety of radiographic studies that attempt to quantify cerebral blood flow and vasoreactivity to identify patients at risk of progression and those who could benefit from surgery. ${ }^{5,6}$ Currently, the reported prognostic value of these assessments varies greatly across institutions, with marked variability in application and interpretation, and few controlled studies. .,5 $^{2}$

Additionally, the rarity of moyamoya poses challenges for radiologists to consistently encounter and grade cases, leading to relatively poor reproducibility between radiological grades among institutions..$^{2,7}$ Inconsistency in assessment based on interpretation of imaging can lead to delays in diagnosis and downstream consequences in clinical management. ${ }^{5}$ New systems are needed in the assessment of this disease group that can both efficiently and accurately give prognostic information to physicians. There is a need to define specific and easily reproducible radiological signatures that can improve risk stratification of patients with moyamoya so that accurate prognostic tools can be applied consistently., 1,3,7,

To address the high variability in current scoring systems and the need for a reliable prognostic tools designed specifically for pediatric moyamoya vasculopathy, our team sought to devise a systematic method to score parenchymal findings on brain $\mathrm{MRI}$ - the pediatric moyamoya MRI score (PMMS) - that can be used easily and in a straightforward manner in clinical decision-making for patients with moyamoya disease and moyamoya syndrome undergoing surgery. The goal when designing the PMMS was to create a system to predict clinical outcomes after surgical treatment of moyamoya. The grading system was constructed using findings easily identified on standard imaging and applied to a cohort of pediatric patients.

\section{Methods}

\section{Patient Cohort and Imaging}

This study was approved by the University of California, San Francisco (UCSF), IRB. Consecutive patients were identified from a UCSF registry of revascularization procedures performed between 2006 and 2021. Inclusion criteria were 1) a diagnosis of moyamoya disease or syndrome as confirmed by imaging and clinical review, and 2) age $\leq 18$ years at the time of the initial surgery. Data were collected retrospectively from the UCSF electronic medical record, including demographics, clinical risk factors, initial presentation, neuroimaging features, treatment, pre- and postoperative stroke events, pediatric stroke outcomes measure (PSOM) score, and modified Rankin Scale (mRS) score preoperatively and at the last follow-up.

A transient ischemic attack (TIA) was defined as neurological dysfunction in a vascular territorial pattern with symptoms lasting $<24$ hours. Ischemic stroke was defined as acute onset, persistence of focal neurological deficit $>24$ hours, and neuroimaging findings consistent with ischemic stroke. Preoperative Suzuki staging, bypass patency, and radiographic outcomes at the last follow-up (reported stable vs worse) were assessed blinded to other clinical variables and outcomes; consensus scores and outcomes were reported.

\section{Grading System}

Radiological variables were chosen for the PMMS based on easily defined parenchymal findings on brain MRI which could be rapidly assessed and used to make a numeric score. Variables with existing correlations between outcomes in moyamoya or other vascular diseases were chosen. $^{9-12}$ Images were scored from 0 to 5 and included ischemic changes $(0-2 ; 0=$ none, $1=$ focal, $2=$ diffuse $)$, encephalomalacia $(0-2 ; 0=$ none, $1=$ focal, $2=$ diffuse $)$, and hemorrhage $(0-1 ; 0=$ not present, $1=$ present $)$ used in the final scoring system; a summary of the PMMS grading system is found in Table 1. Diffuse findings were defined as those present bilaterally or spanning multiple vascular territories. Images used for scoring included the following: T1-weighted, T2-weighted, T2-weighted FLAIR, susceptibility weighted imaging (SWI), and gradient echo sequences. Grading using the PMMS was applied to images obtained within 18 months of the patient's first surgery and was correlated with PSOM and mRS scores preoperatively and at the last follow-up. All images were scored blinded to other clinical variables and outcomes. An example of the PMMS system applied to 2 patients with moyamoya vasculopathy is displayed in Fig. 1.

\section{Surgical Technique}

The preferred surgical approach used in this cohort was a combined direct bypass with indirect revascularization if there was an available superficial temporal artery (STA) donor vessel and large enough cortical recipient branch ( $\geq 1 \mathrm{~mm}$ in diameter). Selection of the surgical approach 

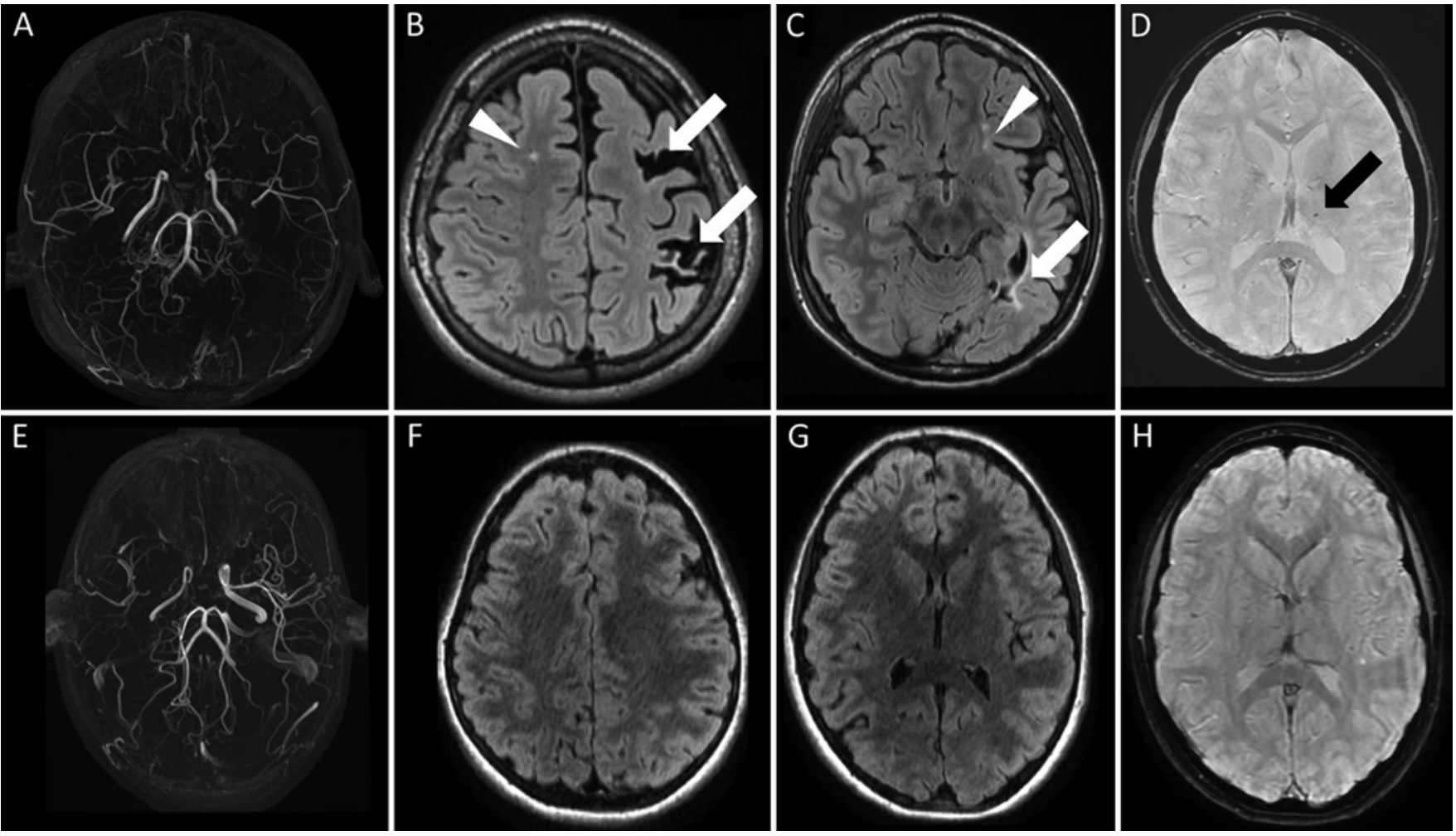

FIG. 1. The PMMS system applied to 2 patients with moyamoya disease. Patient 1 (A-D) had a high PMMS of 5 and patient 2 $(\mathrm{E}-\mathrm{H})$ had a low PMMS of 0 . A: Axial maximal intensity projection (MIP) image of a time-of-flight MRA demonstrates severe narrowing of bilateral MCAs, particularly involving the $M_{1}$ and bilateral ACAs. B and C: Axial FLAIR images at two different levels demonstrate bilateral ischemic changes (white arrowheads indicate a score of 2) and extensive left-sided encephalomalacia (white arrows indicate a score of 2). D: Punctate microhemorrhage is present in the left thalamus (black arrow indicates a score of 1). E: Axial MIP image of time-of-flight MRA demonstrates narrowing of the right internal carotid artery and right MCA, particularly affecting the $M_{1}$ and bilateral ACAs. F and G: Axial FLAIR images at two different levels demonstrate no ischemic changes or encephalomalacia. H: Axial SWI demonstrates no evidence of hemorrhage.

took into consideration severity and frequency of clinical symptoms, with a direct bypass favored for patients with acutely progressive disease. For combined direct and indirect bypass, the following technique was used. After harvesting both the frontal and parietal STA branches when available, the more prominent branch was chosen for direct anastomosis. A small craniotomy overlying the middle cerebral artery (MCA) territory was performed. The STA lumen was widened and sutured with continuous 10-0 sutures to a cortical MCA branch in an end-to-side technique. Bypass patency was confirmed intraoperatively with indocyanine green video angiography. All patients were administered a daily aspirin after being diagnosed with moyamoya, which continued through surgery. After the direct bypass, encephalomyosynangiosis was performed. The arachnoid mater was opened, and the temporalis muscle was split in half in its sagittal plane, with the inner portion used as an indirect onlay graft on the cortex. After replacing the bone flap, the outer portion of muscle was reapproximated with the muscle cuff at the superior temporal line.

For indirect bypass alone, the following techniques were used. After harvesting the STA branches, a small craniotomy was performed overlying the MCA territory. After opening the dura mater, the STA was secured with a
9-0 suture to the pial surface of the brain and, in addition, the inner leaflet of the split temporalis muscle was placed over the exposed brain surface. In some cases (depending on surgeon preference and anatomical limitations), the temporalis muscle was not split and was secured to the surface of the brain.

\section{Outcome Measures}

Outcomes were measured using the PSOM and pediatric $\mathrm{mRS}$ scores through retrospective chart review from neurological examinations documented preoperatively and at the last follow-up. These scoring systems have been validated previously in pediatric populations. ${ }^{13,14}$ Higher values in both scoring systems reflect greater disability.

\section{Statistical Analysis}

Statistical analysis was performed using $\mathrm{R}$ version 3.5.1 (The R Foundation), and graphs were created with GraphPad Prism (GraphPad Software, Inc.). Descriptive statistics were used to define the patient cohort, including patient demographics, moyamoya etiology, preoperative Suzuki stage, treatment details, and clinical and imaging outcomes. Pearson correlation analysis was used to compare the degree of correlation between clinical and im- 
TABLE 2. Clinical and demographic characteristics of the patient cohort

\begin{tabular}{|c|c|c|}
\hline Demographics & \multicolumn{2}{|c|}{ Value $(n=35)$} \\
\hline Mean age at diagnosis, yrs & \multicolumn{2}{|c|}{$8.4 \pm 4.7$} \\
\hline Mean age at 1st surgery & \multicolumn{2}{|c|}{$12.6 \pm 4.6$} \\
\hline \multicolumn{3}{|l|}{ Sex } \\
\hline $\mathrm{F}$ & \multicolumn{2}{|c|}{$22(63)$} \\
\hline M & \multicolumn{2}{|c|}{$13(37)$} \\
\hline Median follow-up, yrs & \multicolumn{2}{|c|}{2.6} \\
\hline Clinical Grading & Preop & Last Follow-Up \\
\hline \multicolumn{3}{|l|}{ mRS score } \\
\hline 0 & $0(0)$ & $9(26)$ \\
\hline 1 & $19(54)$ & $12(34)$ \\
\hline 2 & $7(20)$ & $5(14)$ \\
\hline 3 & $6(17)$ & $5(14)$ \\
\hline 4 & $3(9)$ & $1(3)$ \\
\hline 5 & $0(0)$ & $0(0)$ \\
\hline 6 & $0(0)$ & $3(9)$ \\
\hline \multicolumn{3}{|l|}{ PSOM score } \\
\hline $0-2$ & $29(83)$ & $26(74)$ \\
\hline $3-4$ & $3(9)$ & $7(20)$ \\
\hline $5-6$ & $3(9)$ & $1(3)$ \\
\hline $7-8$ & $0(0)$ & $1(3)$ \\
\hline \multirow[t]{2}{*}{$9-10$} & $0(0)$ & $0(0)$ \\
\hline & Rt Hemisphere & Lt Hemisphere \\
\hline \multicolumn{3}{|l|}{ Preop Suzuki stage } \\
\hline 1 & $0(0)$ & $0(0)$ \\
\hline II & $10(29)$ & $10(29)$ \\
\hline III & $12(34)$ & $9(26)$ \\
\hline IV & $5(14)$ & $11(31)$ \\
\hline V & $4(11)$ & $4(11)$ \\
\hline \multirow[t]{2}{*}{$\mathrm{VI}$} & $0(0)$ & $0(0)$ \\
\hline & \multicolumn{2}{|c|}{ Value } \\
\hline \multicolumn{3}{|l|}{ PMMS } \\
\hline 0 & \multicolumn{2}{|c|}{$14(40)$} \\
\hline 1 & \multicolumn{2}{|c|}{$7(20)$} \\
\hline 2 & \multicolumn{2}{|c|}{$6(17)$} \\
\hline 3 & \multicolumn{2}{|c|}{$4(11)$} \\
\hline 4 & \multicolumn{2}{|c|}{$3(9)$} \\
\hline 5 & \multicolumn{2}{|c|}{$1(3)$} \\
\hline \multicolumn{3}{|l|}{ Disease } \\
\hline MMD & \multicolumn{2}{|c|}{$21(60)$} \\
\hline MMS & \multicolumn{2}{|c|}{$14(40)$} \\
\hline \multicolumn{3}{|l|}{ Presenting symptom } \\
\hline TIA & & \\
\hline Stroke & & 26) \\
\hline Seizure & & 14) \\
\hline Chorea & & 11) \\
\hline Incidental & & 11) \\
\hline
\end{tabular}

》 CONTINUED FROM PREVIOUS COLUMN

TABLE 2. Clinical and demographic characteristics of the patient cohort

\begin{tabular}{lc}
\hline & Value \\
\hline Surgery type & \\
\hline Direct plus indirect & $19(54)$ \\
\hline Indirect alone & $16(46)$ \\
\hline Complication & \\
\hline Stroke & $3(9)$ \\
\hline TIA & $1(3)$ \\
\hline Arterial injury & $1(3)$ \\
\hline Wound infection & $1(3)$ \\
\hline Acute kidney injury & $1(3)$ \\
\hline Involved hemisphere & $1(3)$ \\
\hline Rt & $3(9)$ \\
\hline Lt & $31(89)$ \\
\hline Bilat
\end{tabular}

$\mathrm{MMD}$ = moyamoya disease; $\mathrm{MMS}$ = moyamoya syndrome.

Values represent the number of patients (\%) or mean \pm SD unless indicated otherwise.

aging-based scores. The Fisher r-to-z transformation was used to test for differences between Pearson correlations. Welch's t-test was performed to compare preoperative and follow-up mean mRS scores between high and low PMMS subgroups. Fisher's exact test was used to compare categorical outcome measures between groups. Area under the receiver operating characteristic curve (AUROC) was calculated for the score as a predictor for either good $(0-2)$ or bad (3-6) mRS scores.

\section{Results}

Thirty-five children with 66 affected hemispheres were included in the study. The cohort was $63 \%$ female and had a mean age of $8.4 \pm 4.7$ years $( \pm \mathrm{SD})$ at diagnosis and 12.6 \pm 4.6 years at the first surgery. Twenty-one patients $(60 \%)$ in the cohort had primary moyamoya disease and 14 patients $(40 \%)$ had moyamoya syndrome. The median clinical follow-up for the cohort was 2.6 years from the date of surgery.

The most common presenting symptom for patients was TIA $(13,37 \%)$, followed by stroke $(9,26 \%)$, seizure $(5,14 \%)$, chorea $(4,11 \%)$, and incidental $(4,11 \%)$. Nineteen patients $(54 \%)$ were treated with a combination of direct and indirect revascularization, and $16(46 \%)$ were treated with indirect revascularization alone. A comprehensive list of clinical and demographic variables assessed in the study is found in Table 2.

By Pearson correlation analysis, PMMS based on preoperative MRI had strong correlations with the mRS score at diagnosis $(\mathrm{r}=0.57,95 \%$ CI $0.29-0.77 ; \mathrm{p}<0.001)$ and at the last follow-up ( $\mathrm{r}=0.7,95 \%$ CI $0.44-0.84 ; \mathrm{p}<0.001)$. A more modest but significant correlation was found with PSOM scores preoperatively $(\mathrm{r}=0.44,95 \%$ CI $0.11-0.68$; $\mathrm{p}=0.01)$ and at the last follow-up $(\mathrm{r}=0.47,95 \% \mathrm{CI} 0.16-$ $0.7 ; p=0.005)$. There was no correlation found between 

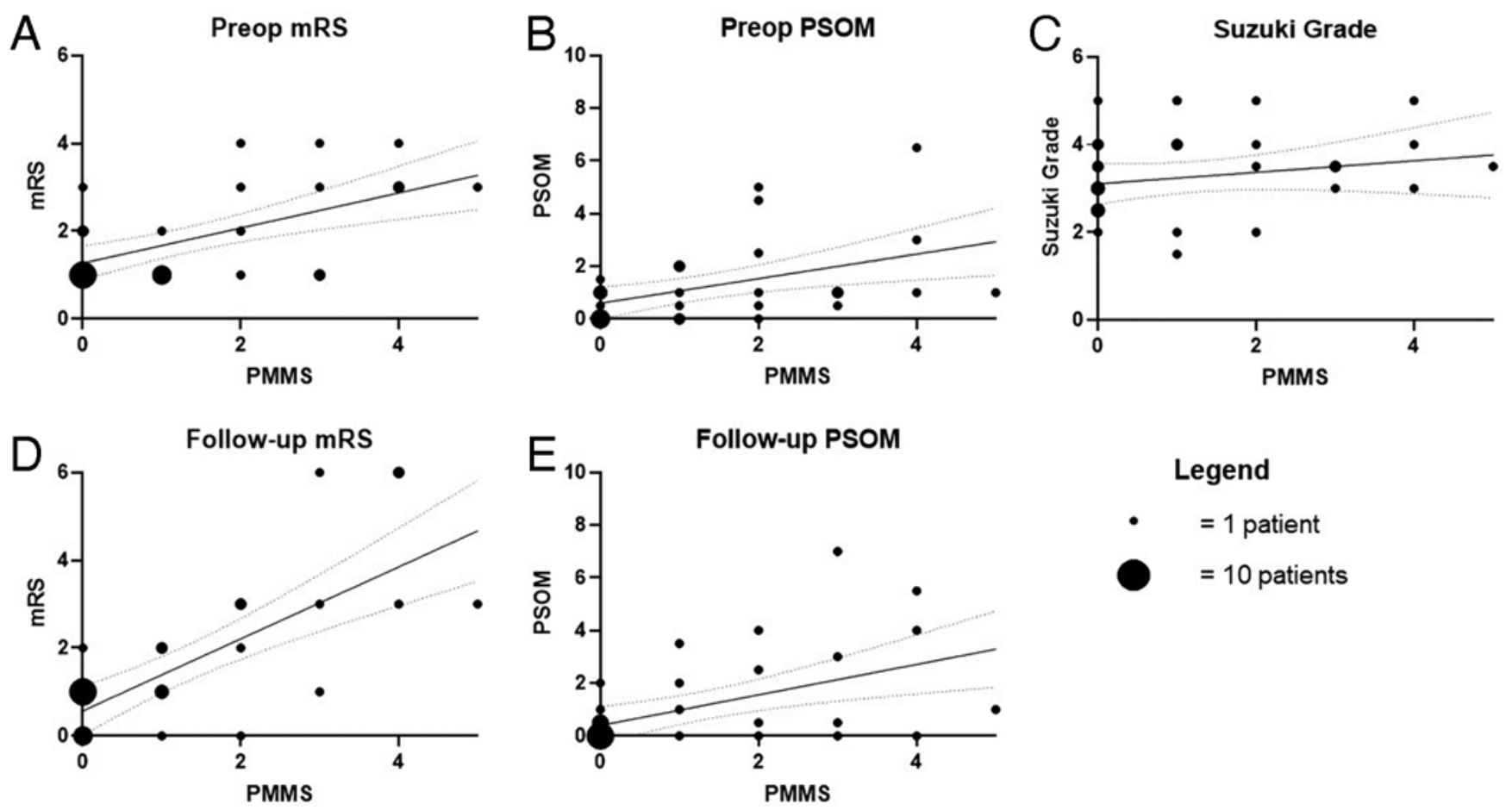

FIG. 2. Pearson correlation analysis of PMMSs with preoperative (A) and follow-up (D) mRS scores, preoperative (B) and followup (E) PSOM scores, and preoperative Suzuki stages (C). Points on the graphs are weighted based on the number of patients. The dotted lines indicate the $95 \% \mathrm{Cl}$ and the solid line indicates the mean.

PMMSs and Suzuki stage $(\mathrm{r}=0.19,95 \% \mathrm{CI}-0.17$ to 0.5 ; $\mathrm{p}=0.3$ ). The Pearson correlation analysis for these groups is shown in Fig. 2. No correlation was found between the Suzuki stage and clinical outcome measure either preoperatively or at the last follow-up.

To control for the potential effects of a diagnosis of moyamoya disease versus syndrome, type of surgery (direct plus indirect revascularization vs indirect revascularization alone), and postoperative clinical stroke on patient outcomes, the Fisher r-to-z transformation was used to test for the effect dichotomization of these variables had on the PMMS correlation with clinical outcomes. Discrimination resulted in no significant difference in correlation controlling for diagnosis of moyamoya disease $(\mathrm{p}=0.48$ preoperative, $p=0.16$ follow-up), type of surgery ( $p=0.15$ preoperative, $p=0.4$ follow-up), and the presence of stroke on postoperative imaging $(p=0.24)$. These results led us to refrain from discriminating between patients in these groups in downstream analysis. A collective list of the Pearson correlation analysis performed is shown in Table 3.

As mRS scores preoperatively and at the last followup held the strongest correlation with the PMMS, further analysis was performed to assess the association between these two scales. The PMMS was sorted into favorable $(0-2)$ and unfavorable (3-5) scores, and the mean mRS scores for both groups were calculated. The mean mRS score of the unfavorable PMMS group was higher in both the preoperative ( $1.5 \mathrm{vs} 2.7, \mathrm{p}=0.02)$ and last follow-up categories (1.0 vs $3.2, \mathrm{p}=0.02)$, with a greater difference observed at the last follow-up (Fig. 3A). Additionally, the mean change in mRS score from preoperatively to follow-
TABLE 3. Pearson correlation analysis for clinical and imaging scores

\begin{tabular}{|c|c|c|c|}
\hline & r & $95 \% \mathrm{Cl}$ & $p$ Value \\
\hline \multicolumn{4}{|l|}{ Variable correlated w/ PMMS } \\
\hline Preop mRS score & 0.58 & 0.3 to 0.77 & $<0.001$ \\
\hline Follow-up mRS score & 0.72 & 0.5 to 0.84 & $<0.001$ \\
\hline Preop PSOM score & 0.44 & 0.11 to 0.68 & 0.01 \\
\hline Follow-up PSOM score & 0.49 & 0.19 to 0.7 & 0.003 \\
\hline Suzuki stage & 0.18 & -0.15 to 0.49 & 0.28 \\
\hline \multicolumn{4}{|l|}{ Preop mRS score } \\
\hline MMD & 0.61 & 0.24 to 0.83 & 0.003 \\
\hline MMS & 0.59 & 0.09 to 0.85 & 0.03 \\
\hline \multicolumn{4}{|l|}{ Follow-up mRS score } \\
\hline MMD & 0.63 & 0.28 to 0.84 & 0.002 \\
\hline MMS & 0.82 & 0.5 to 0.94 & $<0.001$ \\
\hline Direct-indirect bypass & 0.73 & 0.43 to 0.89 & $<0.001$ \\
\hline Indirect bypass alone & 0.68 & 0.27 to 0.89 & 0.005 \\
\hline Excluding postop stroke & 0.59 & 0.3 to 0.79 & $<0.001$ \\
\hline \multicolumn{4}{|c|}{ Variable correlated w/ Suzuki stage } \\
\hline Preop mRS score & 0.08 & -0.26 to 0.4 & 0.64 \\
\hline Follow-up mRS score & 0.08 & -0.26 to 0.4 & 0.64 \\
\hline Preop PSOM score & 0.04 & -0.3 to 0.37 & 0.83 \\
\hline Follow-up PSOM score & 0.05 & -0.29 to 0.37 & 0.79 \\
\hline
\end{tabular}



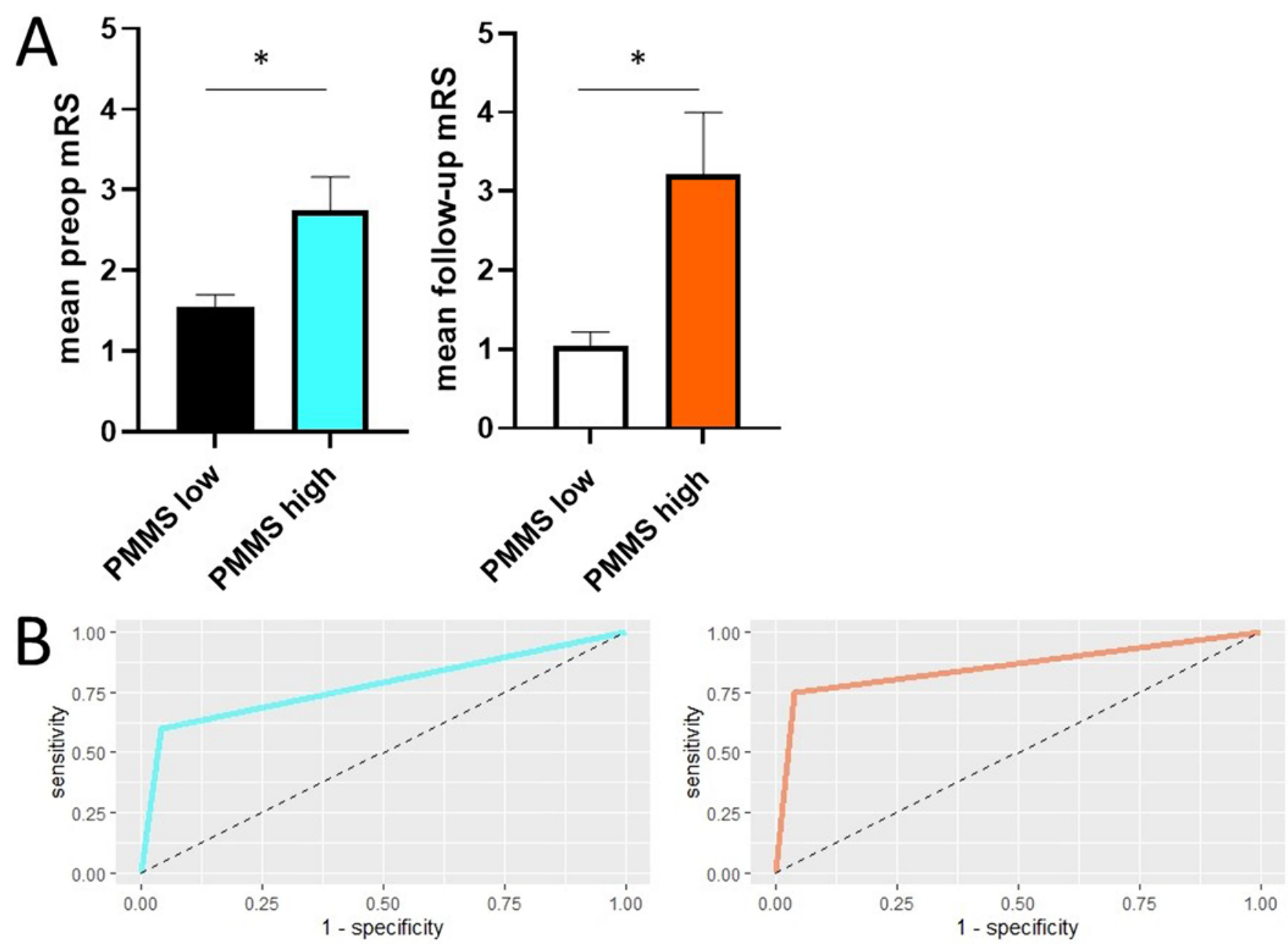

FIG. 3. Preoperative and follow-up mRS scores based on high or low PMMSs. A: Comparison of preoperative and follow-up mean mRS scores between low (favorable) and high (unfavorable) PMMS subgroups. B: AUROC calculated for PMMS as a predictor for either good (0-2) or bad (3-6) mRS scores. * $p<0.05$, Welch's t-test.

up was found to be significantly higher in the unfavorable PMMS group ( $0.67 \mathrm{vs}-0.48, \mathrm{p}=0.01)$, meaning functional status worsened for patients with high PMMSs while it improved for patients with low PMMSs. No association was found between high PMMSs and intraoperative complications ( $\mathrm{p}=0.58$, Fisher's exact test). Preoperative and follow-up mRS scores were then stratified into good (0-2) and bad (3-6), and the AUROC was calculated to gain further insight into the predictive value of this score in this cohort, yielding an AUROC of 0.77 for a high preoperative $\mathrm{mRS}$ score and 0.85 for a high follow-up mRS score (Fig. 3B).

\section{Discussion}

Assessment of patients with moyamoya using a scoring system based on MRI holds several advantages over other imaging classification methods., ${ }^{5,15}$ MRI is readily available at most pediatric medical centers, and radiologists are familiar with interpretation of the images. DSA data can be more challenging to interpret, given differences in technique during invasive testing, and scoring systems may vary greatly across institutions and providers, which is further compounded by the rarity of moyamoya. ${ }^{2,16} \mathrm{In}$ addition, follow-up angiography is not routinely available, and thus MRI represents a more suitable comparison. Perfusion-based scans have been shown to correlate well with angiographic scoring of moyamoya disease, are often relative instead of absolute in their assessment of blood flow, and only give information for the brain's perfusion status at a single time point. ${ }^{17,18}$ Nuclear medicine techniques such as PET and SPECT have shown some promise in pilot studies but have poor spatial and temporal resolution and involve the administration of radioactive tracers, some of which have very short half-lives, thus rendering the studies impractical unless there is an on-site cyclotron..$^{19,20}$ The PMMS has the potential to be a clinically useful and easily applied tool in the diagnosis and treatment of pediatric moyamoya.

In this small cohort of pediatric patients, a correlation was found between preoperative PMMSs and mRS scores both preoperatively and at the last follow-up (Table 3). A weak, although still significant, correlation with the PSOM 


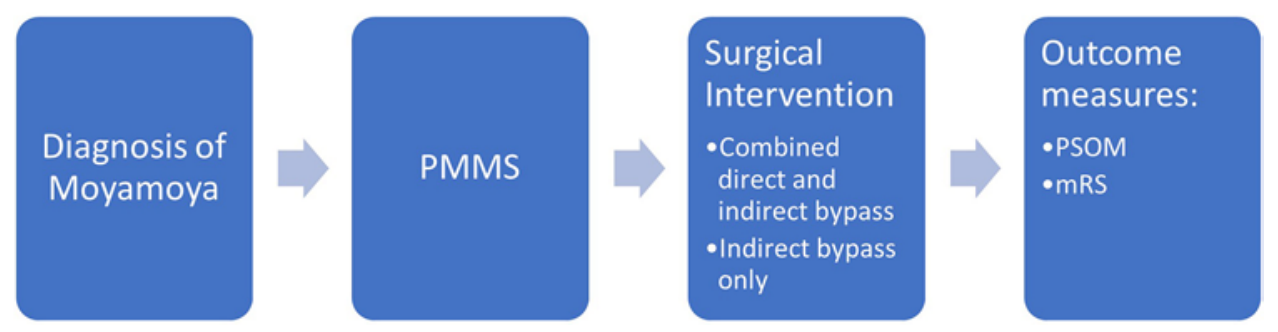

FIG. 4. Flowchart demonstrating that the PMMS applied preoperatively is correlated with postoperative outcome measures (PSOM and $\mathrm{mRS}$ scores).

score was found. The correlation between PMMSs and mRS scores on follow-up suggests that the PMMS scoring system may be used to better inform patients of their prognosis following surgical treatment for moyamoya. A flowchart demonstrating the potential use of the PMMS system in a clinical setting is displayed in Fig. 4.

The PMMS is a straightforward grading system that correlates with postoperative disability in children with moyamoya vasculopathy after surgical treatment, regardless of treatment modality. The simplicity of the PMMS, which is based on easily interpretable MRI findings, allows for rapid calculation and smooth adoption into the workflow of moyamoya management. The key benefit of this score is its potential for stratifying clinical outcome after surgery compared with currently employed grading scales. Surgeons, as well as patients and their families, may be willing to move forward with surgery more readily if a scoring system such as the PMMS can predict that the patient will have a favorable outcome. The combination of simplicity and accuracy makes the PMMS a promising tool to predict outcome following surgical treatment of moyamoya. This scale is intended to be used as a piece of the larger clinical picture for decision-making by physicians and is not meant to supersede clinical judgment.

While this retrospective study yielded promising results, future prospective studies in larger cohorts, at multiple treatment centers, and in adult populations will be needed to determine whether these results can be generalized on a larger scale. Clinical scales scored at multiple time points should also be taken to stratify risk over time and to see if there is an optimal time frame in which this score can be used to predict clinical outcomes. Finally, further studies should include other treatment modalities to see if these affect the performance of the scoring system.

The results of this study should be interpreted in the context of several limitations. Most notably, this study was conducted in a relatively small cohort from a single institution. Thus, sampling bias may have occurred, and more nuanced associations seen in larger multicenter studies may have gone undetected. Second, the clinical grades correlated with the PMMS (mRS and PSOM scores) were taken at only two time points and were ascertained from chart review and, therefore, may be limited by variability in examination or documentation by the treating clinicians. Third, it is possible that components of the PMMS (i.e., ischemia and hemorrhage) may have influenced decision-making by physicians and eventual patient outcomes. Finally, this study is unable to directly determine if ap- plying the PMMS prospectively will enhance diagnostic accuracy or influence treatment algorithms; however, the value of this score is based on its ability to combine information from standardized imaging components to potentially provide clinicians with more precise estimates of the probability outcomes for patients with moyamoya.

\section{Conclusions}

The PMMS is a simple tool based on preoperative MRI data that can be quickly and easily calculated and can potentially aid in the prediction of outcomes and clinical decision-making for patients with moyamoya disease and moyamoya syndrome undergoing surgical treatment. Future prospective studies are needed to assess the generalizability of this scoring system.

\section{References}

1. Kuroda S, Houkin K. Moyamoya disease: current concepts and future perspectives. Lancet Neurol. 2008;7(11):10561066.

2. Scott RM, Smith ER. Moyamoya disease and moyamoya syndrome. N Engl J Med. 2009;360(12):1226-1237.

3. Smith ER, Scott RM. Moyamoya: epidemiology, presentation, and diagnosis. Neurosurg Clin N Am. 2010;21(3):543-551.

4. Hervé D, Ibos-Augé N, Calvière L, Rogan C, Labeyrie MA, Guichard JP, et al. Predictors of clinical or cerebral lesion progression in adult moyamoya angiopathy. Neurology. 2019; 93(4):e388-e397.

5. Yeon JY, Shin HJ, Kong DS, Seol HJ, Kim JS, Hong SC, Park $\mathrm{K}$. The prediction of contralateral progression in children and adolescents with unilateral moyamoya disease. Stroke. 2011; 42(10):2973-2976.

6. Kelly ME, Bell-Stephens TE, Marks MP, Do HM, Steinberg GK. Progression of unilateral moyamoya disease: a clinical series. Cerebrovasc Dis. 2006;22(2-3):109-115.

7. Kim JE, Jeon JS. An update on the diagnosis and treatment of adult moyamoya disease taking into consideration controversial issues. Neurol Res. 2014;36(5):407-416.

8. Funaki T, Takahashi JC, Takagi Y, Kikuchi T, Yoshida K, Mitsuhara T, et al. Unstable moyamoya disease: clinical features and impact on perioperative ischemic complications. J Neurosurg. 2015;122(2):400-407.

9. Nag C, Das K, Ghosh M, Khandakar MR. Prediction of clinical outcome in acute hemorrhagic stroke from a single CT scan on admission. N Am J Med Sci. 2012;4(10):463-467.

10. Ryan RWJ, Chowdhary A, Britz GW. Hemorrhage and risk of further hemorrhagic strokes following cerebral revascularization in moyamoya disease: a review of the literature. Surg Neurol Int. 2012;3:72.

11. Kirton A, Deveber G, Pontigon AM, Macgregor D, Shroff M. 
Presumed perinatal ischemic stroke: vascular classification predicts outcomes. Ann Neurol. 2008;63(4):436-443.

12. Kuroda S, Hashimoto N, Yoshimoto T, Iwasaki Y. Radiological findings, clinical course, and outcome in asymptomatic moyamoya disease: results of multicenter survey in Japan. Stroke. 2007;38(5):1430-1435.

13. Engelmann KA, Jordan LC. Outcome measures used in pediatric stroke studies: a systematic review. Arch Neurol. 2012; 69(1):23-27.

14. Kitchen L, Westmacott R, Friefeld S, MacGregor D, Curtis $\mathrm{R}$, Allen A, et al. The pediatric stroke outcome measure: a validation and reliability study. Stroke. 2012;43(6):1602-1608.

15. Li J, Jin M, Sun X, Li J, Liu Y, Xi Y, et al. Imaging of moyamoya disease and moyamoya syndrome: current status. $J$ Comput Assist Tomogr. 2019;43(2):257-263.

16. Bacigaluppi S, Dehdashti AR, Agid R, Krings T, Tymianski $\mathrm{M}$, Mikulis DJ. The contribution of imaging in diagnosis, preoperative assessment, and follow-up of moyamoya disease: a review. Neurosurg Focus. 2009;26(4):E3.

17. Calamante F, Ganesan V, Kirkham FJ, Jan W, Chong WK, Gadian DG, Connelly A. MR perfusion imaging in moyamoya syndrome: potential implications for clinical evaluation of occlusive cerebrovascular disease. Stroke. 2001;32(12): $2810-2816$

18. Togao O, Mihara F, Yoshiura T, Tanaka A, Noguchi T, Kuwabara Y, et al. Cerebral hemodynamics in moyamoya disease: correlation between perfusion-weighted MR imaging and cerebral angiography. AJNR Am J Neuroradiol. 2006; 27(2):391-397.
19. Kuhn FP, Warnock G, Schweingruber T, Sommerauer M, Buck A, Khan N. Quantitative H2[(15)O]-PET in pediatric moyamoya disease: evaluating perfusion before and after cerebral revascularization. J Stroke Cerebrovasc Dis. 2015; 24(5):965-971.

20. Volkan-Salanci B, Lay Ergün E, Genc Sel Ç, Yalnizoğlu D, Turanli G. The role of brain perfusion SPECT in moyamoya disease. Rev Esp Med Nucl Imagen Mol. 2012;31(4):216-218.

\section{Disclosures}

The authors report no conflict of interest concerning the materials or methods used in this study or the findings specified in this paper.

\section{Author Contributions}

Conception and design: Hetts, Garcia, Morshed. Acquisition of data: Garcia, Morshed, Winkler, Li, Fox, Rutledge, Beniwal. Analysis and interpretation of data: Hetts, Garcia, Li. Critically revising the article: Hetts, Morshed, Winkler, Li, Fox, Fullerton, Lawton, Abla, Gupta. Reviewed submitted version of manuscript: all authors. Statistical analysis: Garcia.

\section{Correspondence}

Steven W. Hetts: University of California, San Francisco, CA. steven.hetts@ucsf.edu. 